\title{
Segurança alimentar e riscos associados à temperatura de preparações quentes em restaurantes comerciais
}

Food safety and associated risks the temperature of hot preparations in commercial restaurants

\author{
E. G. D. S. Cardozo ${ }^{1}$; F. M. Gottardo ; K. B. Sartor ${ }^{3}$; C. D. S. Peixoto ${ }^{1}$; A. L. S. \\ Alves $^{4}$; L. B. Rodrigues ${ }^{1}$; L. R. Santos ${ }^{1,2 *}$

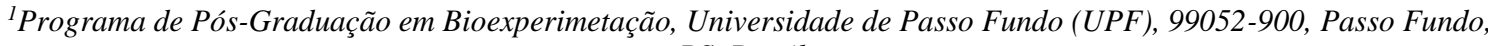 \\ RS, Brasil. \\ ${ }^{2}$ Programa de Pós-Graduação em Ciência e Tecnologia de Alimentos, Universidade de Passo Fundo (UPF), 99052 - \\ 900, Passo Fundo, RS, Brasil. \\ ${ }^{3}$ Curso de Engenharia de Alimentos, Universidade de Passo Fundo (UPF), 99052-900,Passo Fundo, RS, Brasil. \\ ${ }^{4}$ Programa de Pós-Graduação em Envelhecimento Humano, Universidade de Passo Fundo (UPF), 99052-900, Passo \\ Fundo, RS, Brasil. \\ *luruschel@upf.br
}

(Recebido em 26 de setembro de 2019; aceito em 28 de fevereiro de 2020)

\begin{abstract}
O ritmo de vida acelerado ampliou o interesse em refeições práticas, aumentando a procura por Unidades de Alimentação e Nutrição (UAN). A qualidade e segurança do alimento fornecido está interligada com parâmetros como tempo e temperatura de exposição da refeição, pois a interação desses fatores influencia na multiplicação microbiana. Assim, o objetivo deste trabalho foi avaliar o tempo e temperatura de preparações quentes de UANs para estimar os riscos correlacionados com a inadequação destes parâmetros e percentual de não conformidades conforme as Boas Práticas de Manipulação de Alimentos. Para tanto, foram aferidas as temperaturas de pratos quentes no período de 10 dias, em três UANs, com noventa minutos de intervalo entre cada aferição, totalizando 540 amostras. Os resultados indicaram temperaturas não conformes na UAN $1(58,6 \%)$, UAN 2 (46\%) e UAN 3 (67,8\%). Em relação às Boas Práticas de Manipulação de Alimentos, a UAN 1 apresentou $35,8 \%$ de conformidade e as unidades 2 e $332,1 \%$ de conformidade. Nas unidades avaliadas identificou-se falhas na manutenção das temperaturas preconizadas, indicando a necessidade do estabelecimento de processos que minimizem ou eliminem estas não conformidades. As preparações de carnes, seguido pelas guarnições e massas, apresentaram os maiores índices de não conformidades, com diminuição das temperaturas conforme o tempo de exposição. Conclui-se que esses fatores apresentaram altas porcentagens de não conformidades e para saná-las, cada UAN deve ser analisada individualmente, verificando-se os fatores causadores destas não conformidades, como equipamentos, quantidade exposta no buffet, número de comensais e período de reposição, visando assim realizar ações corretivas nos pontos críticos identificados.

Palavras-chave: Doenças Transmitidas por Alimentos, Boas Práticas, Buffet.
\end{abstract}

The fast pace of life has increased interest in practical meals, increasing the demand for Food and Nutrition Units (UAN). The quality and safety of the food provided is interconnected with parameters such as meal exposure time and temperature, as the interaction of these factors influences microbial multiplication. Thus, the objective of this study was to evaluate the time and temperature of hot UAN preparations to estimate the risks correlated with the inadequacy of these parameters and the percentage of nonconformities according to Good Food Handling Practice. Therefore, hot plate temperatures were measured over 10 days in three UANs, with ninety minutes of interval between each measurement, totaling 540 samples. The results indicated nonconforming temperatures in UAN 1 (58.6\%), UAN 2 (46\%) and UAN 3 (67.8\%). In relation to Good Food Handling Practices, UAN 1 had 35.8\% compliance and units 2 and $332.1 \%$ compliance. In the units evaluated, failures in maintaining the recommended temperatures were identified, indicating the need to establish processes that minimize or eliminate these nonconformities. The meat preparations, followed by the garnishes and pastas, presented the highest nonconformity indexes, with decreasing temperatures according to the exposure time. It is concluded that these factors presented high percentages of nonconformities and to remedy them, each UAN must be analyzed individually, verifying the factors that cause these nonconformities, such as equipment, amount exposed in the buffet, number of diners and replacement period, thus aiming to perform corrective actions at the identified critical points.

Keywords: Foodbone Diseases, Good Manufacturing, Buffet. 


\section{INTRODUÇÃO}

O aumento no ritmo de vida dos indivíduos alterou seus hábitos alimentares, onde fatores como jornada de trabalho, população em centros urbanos e dificuldades de locomoção contribuíram para a alimentação fora do ambiente familiar, ampliando o número de Unidades de Alimentação e Nutrição (UAN). Uma UAN é definida como unidade de trabalho que desempenha atividades de alimentação e nutrição para coletividades, que objetiva o fornecimento de refeições adequadas e seguras em nutrição e sanidade [13].

A qualidade das refeições ofertadas em uma UAN depende de fatores como matéria prima, higiene dos equipamentos e utensílios e cuidado dos manipuladores. Parâmetros como tempo e temperatura estão intimamente relacionados com a segurança do alimento ofertado, visto que a interação desses fatores influencia na multiplicação de microrganismos $[4,11,18]$.

Os alimentos de origem vegetal ou animal, frescos ou processados, incluindo a água, podem veicular diversos microrganismos patogênicos, que causam danos à saúde dos consumidores, desde sintomas leves até enfermidades graves [5].

As contaminações do alimento por esses microrganismos podem ocorrer em qualquer ponto de produção e distribuição, onde a maioria da incidência de Doenças Veiculadas por Alimentos (DVAs) é causada por falhas de boas práticas, como manipulação e preparação inadequada dos alimentos nos serviços de alimentação [19, 21]. Segundo o Informe de Surtos de DVAs do Ministério da Saúde, entre 2009 a 2018, cerca de 37,2\% destes surtos ocorreram nas residências e $16 \%$ em restaurantes/padarias e similares, com destaque para Escherichia coli $(23,4 \%)$ e Salmonella spp. (11,3\%) [3].

Para assegurar que os alimentos produzidos em UANs estejam em condições higiênicosanitárias adequadas, foi regulamentada no Brasil a Resolução da Diretoria Colegiada 216 - RDC 216 [2], que estabelece procedimentos de boas práticas para serviços de alimentação. Essa resolução prevê, por exemplo, que alimentos que passaram por cocção e que serão expostos para consumo em buffets devem ser mantidos a $60^{\circ} \mathrm{C}$ por no máximo 6 horas. Nos serviços de alimentação é estabelecida também a Portaria 78 , de 30 de janeiro de 2009, que estabelece os procedimentos de boas práticas para serviços de alimentação, a fim de garantir as condições higiênico-sanitárias do alimento preparado. Também, a Resolução da Diretoria Colegiada 275, de 21 de outubro de 2002 - RDC 275 [22], estabelece a avaliação dos equipamentos de conservação de alimentos e a existência de registros das temperaturas durante a conservação.

Para assegurar a inocuidade dos alimentos, o binômio tempo e temperatura é um fator importante que deve ser controlado de maneira adequada, para não favorecer a multiplicação de patógenos [9, 34].

Com o exposto, avaliou-se o binômio tempo e temperatura de preparações quentes dispostas em buffets de Unidades de Alimentação e Nutrição a fim de avaliar os riscos correlacionados com o tempo e temperatura inadequados e percentual não conformidades das adequações de itens contemplados nas Boas Práticas de Manipulação de Alimentos.

\section{MATERIAL E MÉTODOS}

Selecionou-se, por conveniência, três Unidades de Alimentação e Nutrição (UANs) empresariais do tipo self-service, localizadas na cidade de Passo Fundo, região norte do Rio Grande do Sul, entre março e maio de 2017, devidamente autorizadas pelas empresas em documento próprio.

Nas unidades amostradas são oferecidas diariamente as preparações arroz, feijão, massa, guarnições e carnes, sendo que na UAN denominada 1 (UAN 1) são ofertados também molhos. As preparações das UANs podem ser refogadas, cozidas, assadas ou fritas.

A UAN 1 oferta cerca de 210 almoços diariamente, cinco vezes por semana, no período entre 11:30h e 14:00h. As preparações são armazenadas em pass through para posterior reposição no buffet, que ocorre uma hora após o início das refeições. A UAN 2 funciona 24 horas, sete vezes por semana, porém somente o almoço para 120 comensais foi contabilizado neste trabalho. A reposição ocorre conforme a demanda e a empresa não dispõe de pass throughs, sendo os alimentos mantidos aquecidos em fogão e/ou forno. A UAN 3 atende 60 comensais diariamente para o almoço, cinco 
vezes por semana, sendo as preparações mantidas em pass through para posterior disposição no buffet mas sem reposição devido ao número reduzido de comensais.

Nas três unidades foram aferidas as temperaturas das preparações quentes na distribuição do almoço, durante 2 semanas de segunda a sexta, totalizando 10 dias, nos tempos inicial, intermediário e final da distribuição, com noventa minutos de intervalo entre cada aferição. $\mathrm{O}$ horário de início da distribuição era caracterizado pela colocação das cubas no buffet. Na UAN1 foram aferidas 210 amostras, na UAN2 150 e na UAN3 180, totalizando 540 aferições de temperaturas. Utilizou-se termômetro digital do tipo "espeto" modelo WT-1, com capacidade de aferição de temperaturas entre $-50^{\circ} \mathrm{C}$ a $300^{\circ} \mathrm{C}$.

Estas UANs também foram avaliadas quanto alguns componentes específicos contidos na Portaria 78 [16], como por exemplo, os itens 9: preparação do alimento; 10: armazenamento e transporte do alimento preparado e 11: exposição ao consumo do alimento preparado [16]. Essa seleção teve como critério a influência desses itens na temperatura final do alimento [3]. A coleta dos dados foi realizada por pesquisador treinado através de observação direta. Cada item de verificação foi avaliado como "conforme" (C: de acordo com o preconizado pela legislação) ou "não conforme" (NC: em desacordo com o preconizado).

Para as variáveis quantitativas foram calculadas as medidas de tendência central e dispersão e para as variáveis qualitativas foram apresentadas as frequências absolutas e relativas simples. Aplicou-se a correlação de Pearson para verificar a correlação entre as variáveis quantitativas, após verificação da normalidade com o teste de Kolmogorov-Smirnov.

\section{RESULTADOS E DISCUSSÃO}

As temperaturas das preparações aferidas nas UANs estudadas mostraram que $57,46 \%$ das amostras não estavam em conformidade com os parâmetros estabelecidos na RDC 216 [2], ou seja, as temperaturas foram inferiores a $60^{\circ} \mathrm{C}$ com diferença significativa entre as temperaturas recomendadas e as verificadas $(\mathrm{p}=0,0003<0,05)$ (Tabela 1$)$.

Tabela 1: Conformidade das temperaturas aferidas em preparações nas Unidade de Alimentação e Nutrição avaliadas (n=540). Passo Fundo (RS), Brasil, 2018.

\begin{tabular}{cccc}
\cline { 2 - 4 } Unidade & Conforme & Não Conforme & Total \\
\hline UAN 1 & $87(41,4 \%)$ & $123(58,6 \%)$ & 210 \\
UAN 2 & $81(54,0 \%)$ & $69(46,0 \%)$ & 150 \\
UAN 3 & $58(32,2 \%)$ & $122(67,8 \%)$ & 18 \\
Total & $226(42,53 \%)$ & $314(57,46 \%)$ & $540(100 \%)$ \\
\hline
\end{tabular}

Segundo a RDC 216, o alimento cozido precisa ser mantido sob temperaturas superiores a $60^{\circ} \mathrm{C}$ para que seja considerado seguro para consumo [2], bem como no Guia de Alimentos e Segurança Sanitária da Agencia Nacional de Vigilância Sanitária [1], onde os alimentos devem ser mantidos sob temperaturas de refrigeração inferiores a $5^{\circ} \mathrm{C}$ ou aquecidos acima de $60^{\circ} \mathrm{C}$. O intervalo compreendido entre essas temperaturas pode ser propício para proliferação microbiológica e, consequentemente, oferecer risco de contaminação, com impacto em Saúde Pública devido as DTAs [1]. A RDC 275 exige dos estabelecimentos o controle em forma de registros dessas temperaturas durante a conservação, sendo avaliado o histórico e cumprimento da legislação [22].

Em relação a UAN 1, as aferições das temperaturas dos alimentos no tempo inicial estavam adequadas à legislação [2]. Porém, as aferições intermediária e final de exposição no buffet mostraram que, com exceção da preparação feijão, as demais não apresentaram temperatura de segurança, ou seja, estavam abaixo de $60^{\circ} \mathrm{C}$ (Tabela 2).

Nos demais resultados da UAN1, verificou-se que as temperaturas intermediárias e finais são semelhantes, estando atreladas a capacidade de manutenção de temperatura do equipamento de distribuição do buffet aquecido. Constatou-se também que os alimentos com temperatura inicial elevada tendem a manter por mais tempo a temperatura preconizada. 
Tabela 2: Aferições de temperaturas $\left(\mathrm{em}{ }^{\circ} \mathrm{C}\right)$, com média e desvio padrão, das preparações na Unidade de Alimentação e Nutrição l (UAN1, n= 210). Passo Fundo (RS), Brasil, 2018.

\begin{tabular}{ccccc}
\cline { 2 - 5 } Preparações & $\begin{array}{c}\text { Temperatura } \\
\text { inicial }\end{array}$ & $\begin{array}{c}\text { Temperatura } \\
\text { intermediria }\end{array}$ & $\begin{array}{c}\text { Temperatura } \\
\text { final }\end{array}$ & $\begin{array}{c}\text { Temperatura } \\
\text { média }\end{array}$ \\
\hline Arroz & $70,20 \pm 7,33$ & $52,20 \pm 7,15$ & $52,30 \pm 6,34$ & $58,23 \pm 10,91$ \\
Feijão & $80,30 \pm 7,70$ & $65,00 \pm 5,29$ & $61,90 \pm 5,55$ & $69,07 \pm 10,18$ \\
Massa & $60,10 \pm 8,06$ & $56,00 \pm 9,12$ & $54,90 \pm 6,69$ & $57,00 \pm 8,06$ \\
Guarnição & $67,10 \pm 17,23$ & $59,10 \pm 6,81$ & $55,30 \pm 6,15$ & $60,50 \pm 11,97$ \\
Molho 1 & $65,30 \pm 5,08$ & $53,30 \pm 5,38$ & $49,30 \pm 8,27$ & $55,97 \pm 9,28$ \\
Molho 2 & $64,20 \pm 6,55$ & $53,80 \pm 4,98$ & $47,60 \pm 4,72$ & $55,20 \pm 8,74$ \\
Carne 1 & $58,00 \pm 13,79$ & $50,10 \pm 12,62$ & $41,80 \pm 8,99$ & $49,97 \pm 13,37$ \\
Média & $66,46 \pm 11,95$ & $55,64 \pm 8,77$ & $51,87 \pm 8,85$ & $57,99 \pm 11,69$ \\
\hline
\end{tabular}

Considerando as temperaturas verificadas diariamente na UAN2, observa-se a conformidade das temperaturas iniciais, mas ao analisar a Tabela 3, verifica-se que as temperaturas intermediárias, após 90 minutos de distribuição estão em desacordo com as temperaturas recomendadas $(53,67 \pm$ $9,92^{\circ} \mathrm{C}$ ) e que, na medição final, após 3 horas, 4 das 5 preparações apresentam conformidades, exceto a carne, com média de $58,50^{\circ} \mathrm{C}$. $\mathrm{O}$ fato de as temperaturas finais apresentarem valores mais altos do que as intermediárias pode ser atribuído à reposição dos alimentos, uma vez que a UAN2 não possui pass through e os alimentos são mantidos aquecidos em panelas sobre fogão ou em forno, conforme a preparação.

Tabela 3: Aferições de temperaturas $\left(\mathrm{em}^{\circ} \mathrm{C}\right)$, com média e desvio padrão, das preparações na Unidade de Alimentação e Nutrição 2 (UAN2, n=150). Passo Fundo (RS), Brasil, 2018.

\begin{tabular}{ccccc}
\cline { 2 - 5 } Preparações & $\begin{array}{c}\text { Temperatura } \\
\text { inicial }\end{array}$ & $\begin{array}{c}\text { Temperatura } \\
\text { intermediria }\end{array}$ & $\begin{array}{c}\text { Temperatura } \\
\text { final }\end{array}$ & $\begin{array}{c}\text { Temperatura } \\
\text { média }\end{array}$ \\
\hline Arroz & $77,83 \pm 5,94$ & $58,47 \pm 8,40$ & $63,14 \pm 8,10$ & $66,48 \pm 11,12$ \\
Feijão & $76,35 \pm 6,91$ & $57,33 \pm 10,21$ & $61,24 \pm 16,40$ & $64,97 \pm 14,15$ \\
Massa & $60,75 \pm 8,30$ & $55,61 \pm 8,43$ & $61,11 \pm 9,12$ & $59,16 \pm 8,70$ \\
Guarnição & $63,44 \pm 15,19$ & $52,38 \pm 6,70$ & $64,57 \pm 10,33$ & $60,13 \pm 12,24$ \\
Carne 1 & $61,03 \pm 6,03$ & $44,57 \pm 10,33$ & $58,50 \pm 8,90$ & $54,70 \pm 11,10$ \\
Média & $67,88 \pm 11,65$ & $53,67 \pm 9,92$ & $61,71 \pm 10,73$ & $61,09 \pm 12,20$ \\
\hline
\end{tabular}

Uma pesquisa realizada em Florianópolis (SC) encontrou $50 \%$ de inconformidades nas temperaturas da distribuição [29]. Assim como Silva e Cardoso (2008) [24], em uma Unidade de Alimentação e Nutrição na cidade de Montes Claros (MG), ao demostrarem que as temperaturas no balcão de distribuição dos pratos quentes tinham um elevado percentual de amostras fora do padrão. Também Silva et al. (2016) [17] indicaram que as temperaturas médias dos alimentos servidos nos restaurantes self service da cidade de Itapaci (GO) estavam inadequadas com relação a legislação vigente. Da mesma forma, Correa et al. (2017) [33] avaliaram 1000 refeições e encontraram temperaturas médias iniciais na distribuição acima de $60^{\circ} \mathrm{C}$, mas que no transcorrer do atendimento reduziram até temperaturas inadequadas

Corroborando com o estudo realizado por Ventimiglia e Basso (2008) [23], o arroz e o feijão são as preparações que apresentaram temperaturas adequadas. Entretanto, a temperatura da carne iniciou adequadamente, mas ao término da distribuição apresentou temperatura inferior à recomenda pela ANVISA [2].

Na UAN3 (Tabela 4), dentre as temperaturas médias verificadas, observou-se que somente a preparação feijão apresentou valores em conformidade com a legislação [2]. Além disso, destacase a baixa adequação da temperatura no tempo inicial das preparações. O fato de a UAN3 ter média de não conformidades, superior as demais unidades, pode ser atribuído ao número de comensais consideravelmente menor que os demais, sendo que na UAN3 as cubas utilizadas na distribuição dos alimentos são rasas $(10 \mathrm{~cm})$ e utilizadas do começo ao fim da exposição no buffet. 
Tabela 4: Aferições de temperaturas ( $\left.\mathrm{em}{ }^{\circ} \mathrm{C}\right)$, com média e desvio padrão, das preparações na Unidade de Alimentação e Nutrição 3 (UAN3, n=180). Passo Fundo (RS), Brasil, 2018.

\begin{tabular}{ccccc}
\cline { 2 - 5 } Preparações & $\begin{array}{c}\text { Temperatura } \\
\text { inicial }\end{array}$ & $\begin{array}{c}\text { Temperatura } \\
\text { intermediria }\end{array}$ & $\begin{array}{c}\text { Temperatura } \\
\text { final }\end{array}$ & $\begin{array}{c}\text { Temperatura } \\
\text { média }\end{array}$ \\
\hline Arroz & $60,20 \pm 9,31$ & $53,50 \pm 8,73$ & $49,30 \pm 6,40$ & $54,33 \pm 9,17$ \\
Feijão & $80,80 \pm 11,31$ & $67,80 \pm 11,17$ & $63,70 \pm 10,60$ & $70,77 \pm 12,97$ \\
Massa & $52,90 \pm 10,72$ & $54,10 \pm 10,87$ & $47,80 \pm 7,60$ & $51,60 \pm 9,90$ \\
Guarniçãa & $58,50 \pm 16,65$ & $52,80 \pm 15,37$ & $49,10 \pm 15,26$ & $53,47 \pm 15,72$ \\
Carne 1 & $55,60 \pm 8,77$ & $50,50 \pm 8,96$ & $46,40 \pm 7,79$ & $50,83 \pm 9,07$ \\
Carne 2 & $53,50 \pm 15,89$ & $52,10 \pm 14,30$ & $47,00 \pm 12,23$ & $50,87 \pm 14,01$ \\
Média & $60,25 \pm 15,34$ & $55,13 \pm 12,73$ & $50,55 \pm 11,67$ & $55,31 \pm 13,84$ \\
\hline
\end{tabular}

As preparações que lideraram os percentuais de não conformidades (NC) nas unidades avaliadas foram as carnes, seguido por guarnições e massas, verificando-se geralmente decréscimo nas temperaturas conforme o tempo de exposição.

Em estudo de Monteiro et al. (2014) [11], a média de temperatura aferida nos alimentos foi $56,0^{\circ} \mathrm{C}( \pm 7,1)$, com variação entre os estabelecimentos pesquisados, sendo a ausência de controle da temperatura e equipamentos inadequados de aquecimento os fatores que mais contribuíram para estas variações. Para garantir a segurança dos alimentos, por meio de temperaturas adequadas na distribuição, é necessário o controle desse fator através do monitoramento constante [32]. Outros fatores importantes para contaminação dos alimentos seriam falta de mão de obra treinada e espaço físico $[10,12,14]$.

Especificamente na UAN1, os alimentos ficavam armazenados a mais de $70^{\circ} \mathrm{C}$ em um pass through. Entretanto, a manutenção dessa temperatura não foi efetiva, visto que somente na preparação feijão as aferições iniciais, intermediárias e finais encontravam-se sob temperatura de segurança conforme a legislação [2]. Da mesma forma, Souza et al. (2019) [30] encontraram 100\% de concordância nas temperaturas do arroz/feijão enquanto nas guarnições o índice de adequações variou entre 0\% a 66,7\% No estudo de Rocha et al. (2019) [31] o feijão foi a única preparação com temperatura média superior à legislação na primeira aferição, sendo que na segunda aferição nenhuma preparação atingiu temperaturas consideradas conformes

A temperatura de exposição da carne (Tabela 1) está relacionada com sua forma de preparo e apresentação no buffet, uma vez que cortes finos em forma de bifes tendem a reter menos calor que preparações em molho, que contém mais água. Na UAN1 as preparações eram servidas em unidades individuais, contribuindo para a não conformidade das temperaturas. Outra situação que pode ter contribuído para estes resultados é a característica sensorial, uma vez que pratos à base de carne precisam manter suculência quando dispostos para consumo, o que impossibilitaria atingir altas temperaturas no interior do alimento.

As preparações com carnes apresentaram as temperaturas mais baixas na exposição nos buffets, sem atingir a temperatura adequada mesmo no tempo inicial, e tal resultado é preocupante já que produtos cárneos ocupam o quinto lugar no ranking de alimentos identificados como causadores de DVAs $[12,18]$. A denominada "proteína 1" apresentou médias de temperatura inferiores ao recomendado pela legislação [2] em todas as unidades avaliadas e em todas as aferições. Outros estudos mostram essa relação, onde as carnes dispostas no buffet ficam abaixo da temperatura adequada [29].

A Salmonella spp., frequentemente relatada em surtos, pode se desenvolver em temperaturas entre 7 e $48^{\circ} \mathrm{C}$, com temperatura ótima entre 35 e $37^{\circ} \mathrm{C}$. Quando insuficientemente cozidos, alimentos à base de carne, leite e ovos são os principais veículos de transmissão desse microrganismo [8].

Em estudo realizado por Puhl e Silva (2015) [15], foram selecionadas cinco preparações quentes (arroz, feijão, guarnição e dois tipos de carne), nas etapas de armazenamento do alimento preparado e na distribuição, sendo que todas estavam acima de $65^{\circ} \mathrm{C}$ no armazenamento e $60^{\circ} \mathrm{C}$ por no máximo 6 horas na distribuição. Já em estudo de Nascimento et al. (2017) [12], a guarnição que variou entre farofa, cuscuz e macarrão, apresentou todas as médias abaixo do exigido pela legislação nas três unidades avaliadas. 
O período de exposição do alimento no buffet acarreta muitas vezes perdas de temperatura (Tabela 3). Esta intercorrência pode ser observada mesmo quando as temperaturas iniciais (saída da cozinha central) estavam acima de $60^{\circ} \mathrm{C}$, enquanto nas finais (10 minutos antes do início da distribuição) apresentavam-se abaixo do recomendado [6, 20].

A semelhança deste estudo, Silva et. al. (2016) [17], mostraram valores inferiores aos preconizados pela legislação na preparação carnes em duas UANs avaliadas, o que pode favorecer a proliferação microbiana. Fidélis (2005) [25] avaliou 97 amostras em cinco restaurantes institucionais, onde $15,5 \%$ dos alimentos à base de carne estavam impróprios para consumo humano devido as contagens de microrganismos em não conformidade com a legislação vigente [26]. Já Rocha et al. (2010) [27], ao aplicarem um check list em 15 restaurantes, observaram que as temperaturas do arroz, feijão, prato principal e guarnição estavam adequadas, corroborando com os achados de Dalpubel et al. (2012) [28] que, ao analisarem arroz, feijão, carne, omelete e guarnição, citam temperaturas de $79,5^{\circ} \mathrm{C}, 84,9^{\circ} \mathrm{C}, 70,8^{\circ} \mathrm{C}, 76,7^{\circ} \mathrm{C}$ e $69,3^{\circ} \mathrm{C}$, respectivamente

Com relação a aplicação do check list com itens selecionados da Portaria 78 [16], a UAN1 apresentou $35,8 \%$ de conformidade, a UAN2 $32,1 \%$ e a UAN3 32,1\%. Nas unidades avaliadas identificou-se falhas na manutenção das temperaturas preconizadas, indicando a necessidade do estabelecimento de processos que minimizem ou eliminem as não conformidades. Para tanto, a manutenção preventiva dos equipamentos tem extrema importância, devendo ser aliada à um processo produtivo eficiente, onde temperaturas de segurança sejam alcançadas na cocção do alimento e mantidas durante a exposição deste. Para essa manutenção, as reposições frequentes das preparações com temperaturas adequadas seriam fundamentais para a conformidade do processo.

As Unidades de Alimentação e Nutrição (UAN) apresentam-se como uma alternativa aos novos hábitos dos indivíduos, mas as preparações devem seguir as legislações para oferecer ao consumidor alimentos seguros, observando-se cuidadosamente a distribuição, visando evitar problemas de contaminação do alimento. Os resultados obtidos nas Unidades estudadas permitem inferir que a avaliação das UANs deve ser feita de forma singular devido às particularidades de cada uma, especialmente estruturais, além da disponibilidade de equipamentos, processo de produção, armazenamento e exposição dos alimentos prontos para o cliente. Como exemplo, na UAN3 não foram registradas "não conformidades" superiores as demais unidades (Tabela 1), o que pode ser atribuído ao número reduzido de comensais.

Também, o tempo e a temperatura são fatores essenciais na conservação de refeições, devendo ser registrados e monitorados constantemente. No presente trabalho, ao se correlacionar o percentual de conformidade das temperaturas aferidas nas três UANs com as analisadas no check list da Portaria 78 [16], é possível verificar uma correlação positiva e forte $(\mathrm{R}=0,6600)$, ou seja, à medida que aumentam os itens em conformidade há um aumento da temperatura. As empresas com Boas Práticas de Manipulação de Alimentos implantadas tendem a ter menos falhas na segurança do alimento e servir alimentos com menor risco de contaminações. Por outro lado, a falta da adoção destas Boas Práticas na rotina de uma UAN pode colaborar com falhas no processo produtivo e possibilitar infecções de origem alimentar [7, 17].

\section{CONCLUSÃO}

Os parâmetros tempo e temperatura apresentaram não conformidades nas unidades avaliadas, destacando-se as preparações de carnes, seguidas por guarnições e massas, verificando-se perda de temperatura conforme o tempo de exposição, o que comprometeria a segurança do alimento.

Assim, uma UAN deve ser analisada individualmente, verificando-se os fatores causadores da não conformidade como equipamentos, quantidade exposta no buffet, número de comensais e período de reposição, visando assim realizar ações corretivas nos pontos críticos identificados.

\section{REFERÊNCIAS BIBLIOGRÁFICAS}

1. Brasil Agência Nacional de Vigilância Sanitária (Anvisa). Guia de Alimentos e Vigilância Sanitária (Versão 1.1) [homepage on the Internet]; 2016. Disponível em: http://portal.anvisa.gov.br/resultadode- 
busca?p_p_id=101\&p_p_lifecycle=0\&p_p_state=maximized\&p_p_mode $=v i e w \& p \_p \_c o l \_i d=c o l u m n-$ 1\&p_p_col_count=1\&_101_struts_action=\%2Fasset_publisher\%2Fview_content $\&$ 101_assetEntryId= 395967\&_101_type=document.

2. Brasil. Agência Nacional de Vigilância Sanitária. Resolução RDC nº. 216, de 15 de setembro de 2004. Regulamentos Técnicos sobre Boas Práticas para Serviços de Alimentação. Diário Oficial da União 16 set 2004.

3. Brasil. Ministério da Saúde. Surtos de Doenças Transmitidas por Alimentos no Brasil, Informe 2018. [homepage on the Internet]; (2019). Disponível: http://portalarquivos2.saude.gov.br/images/pdf/2019/fevereiro/15/Apresenta----o-Surtos-DTA--Fevereiro-2019.pdf.

4. Conzatti S, Adami F, Fassina P. Monitoramento do tempo e temperatura de refeições transportadas de uma unidade de alimentação e nutrição. Rev Uningá Rev. 2015; 24(2):7-12.

5. Costa ALS. A Microbiologia dos Alimentos e a Importância dos Microrganismos Úteis, Deteriorantes e patogênicos. Microbiologia e Higiene Alimentar. [Internet]. Universidade Anhembi, Morumbi, 2010. Disponível em: https://alinearantess.files.wordpress.com/2015/04/a-microbiologia-dos-alimentos-e-aimportc3a2ncia-dos-microorganismos-c3bateis-deteriorantes-e-patogc3aanicos.pdf.

6. Devides G, Maffei D, Catanozi M. Perfil socioeconômico e profissional de manipuladores de alimentos e o impacto positivo de um curso de capacitação em Boas Práticas de Fabricação. Braz J Food Technol. 2014;17(2):166-176, doi: 10.1590/bjft.2014.014

7. Djekic I, Smigic N, Kalogianni E, Rocha A, Zamioudi L, Pacheco R. Food hygiene practices in different food establishments. Food Control. 2014;39:34-40, doi: 10.1016/j.foodcont.2013.10.035

8. Flores AMPC, Melo CB. Principais bactérias causadoras de doenças de origem alimentar. Rev Bras Med Vet. 2015;3(1):65-72.

9. Germano PML, Germano MIS. Higiene e Segurança Sanitária de Alimentos. 4. ed. São Paulo: Manole; 2011.

10. Kadariya J, Smith T, Thapaliya D. Staphylococcus aureus and Staphylococcal Food-Borne Disease: An Ongoing Challenge in Public Health. BioMed Res Int. 2014;2014:1-9.

11. Monteiro M, Ribeiro R, Fernandes B, Sousa J, Santos L. Controle das temperaturas de armazenamento e de distribuição de alimentos em restaurantes comerciais de uma instituição pública de ensino. Demetra: Alimentação, Nutrição \& Saúde. 2014;9(1):99-106, doi: 10.12957/demetra.2014.6800

12. Nascimento L, Pontes C, Sousa F, Morais M, Queiroz A. Binômio tempo e temperatura de preparações em serviço de alimentação transportada. Nutrivisa: Rev Nutr Vig Saúde. 2017;3(3), doi: 10.17648/nutrivisa-vol-3-num-3-c

13. Oliveira BG, Pereira GHM, Xavier LF, Niero DM. Análise da elaboração do manual de boas práticas para unidades de alimentação e nutrição. Cad Escola Saúde 2015;1(3).

14. Penedo A, Jesus R, Silva S, Monteiro M, Ribeiro R. Avaliação das temperaturas dos alimentos durante o preparo e distribuição em restaurantes comerciais de Belo Horizonte-MG. Demetra: Alimentação, Nutrição \& Saúde. 2015;10(2),doi: 10.12957/demetra.2015.15317

15. Puhl IC, Silva ABG. Verificação da temperatura de alimentos em uma unidade de alimentação e nutrição do município de Lajedo - RS. Higiene Alimentar 2015;29:72-75.

16. Secretária da Saúde (Rio Grande do Sul). Portaria no. 78, de 28 de janeiro de 2009. Aprova a lista de verificação em boas práticas para serviços de alimentação, aprova normas para cursos de capacitação em boas práticas para serviços de alimentação e dá outras providências Diário Oficial do Estado do Rio Grande do Sul 30 jan 2009.

17. Silva GA, Silva LA, Alves CCM. Temperatura de expositores de alimentos e qualidade higiênico sanitária em restaurantes self-service, na cidade de Itapaci-GO. Rev Eletr Faculd Ceres 2016;5(2).

18. Silva LC, Dos Santos DB, De São José JFB, Da Silva EMM. Boas práticas na manipulação de alimentos em unidades de alimentação e nutrição. Demetra: Alimentação, Nutrição \& Saúde. 2015;10(4),doi: 10.12957/demetra.2015.16721.

19. Smigic N, Djekic I, Martins M, Rocha A, Sidiropoulou N, Kalogianni E. The level of food safety knowledge in food establishments in three European countries. Food Control. 2016;63:187-194,doi: 10.1016/j.foodcont.2015.11.017

20. Werner JB, Zilio R, Pereira FB. Avaliação das boas práticas de fabricação de refeições transportadas em unidade de alimentação e nutrição da Serra Gaúcha. Higiene Alimentar 2016;29(240/241):74-79.

21. World Health Organization (WHO). Food safety [homepage on the Internet]; 2019. Disponível em: https://www.who.int/news-room/fact-sheets/detail/food-safety.

22. Agência Nacional de Vigilância Sanitária (Brasil). Resolução RDC no 275, de 21 de outubro de 2002. Regulamentos Técnicos sobre estabelecimentos produtores/industrializadores de alimentos e lista de verificação das boas práticas de fabricação em estabelecimentos produtores/industrializadores de alimentos. Diário Oficial da União 23 out 2003 
23. Ventimiglia TM, Basso C. Tempo e temperatura na distribuição de preparações em Unidade de Alimentação e Nutrição. Disc Scientia 2008;9(1):109-14.

24. Silva BAF, Cardoso RR. Temperatura de alimentos em uma Unidade de Alimentação e Nutrição do município de Montes Claros - MG. Semana da Biologia 2008.

25. Fidélis GA. Avaliação das boas práticas de preparação em restaurantes institucionais [dissertação]. Viçosa: Universidade Federal de Viçosa; 2005.

26. Ministério da Saúde (Brasil). Resolução RDC no. 12, de 02 de janeiro de 2001. Regulamento técnico sobre padrões microbiológicos para alimentos. Diário Oficial da República Federativa do Brasil 10 jan 2001.

27. Rocha B, Batista Da S, Borges BMA, Paiva AC. Avaliação das condições higiênico-sanitárias e da temperatura das refeições servidas em restaurantes comerciais do tipo self-service. Rev Núcleo Interd Pesq Ext UNIPAM 2010;7(1):30-40.

28. Dalpubel V, Busch L, Giovanoni A. Relação entre alimento seguro e a temperatura de preparações quentes do buffet de uma unidade de alimentação e nutrição no Vale do Taquari, RS. Rev Destaques Acad. 2012;4(3)

29. Silva BB, Boni BR, Schlindwein AD. Tempo de exposição e temperatura de distribuição da refeição quente dos funcionários de uma unidade de alimentação e nutrição hospitalar de Florianópolis-SC. Reva UNINGÁ, Maringá, 2019,56(3):132-140.

30. Sousa AVR, Pereira DM, Pereira CTM. Avaliação da temperatura de preparações servidas em uma Unidade de Alimentação e Nutrição. Res, Soc Develop. 2019;8(5), doi: http://dx.doi.org/10.33448/rsdv8i5.967.

31. Rocha LA, Rodrigues LM, Araújo MC, Soares TC, Gomes SAB, Oliveira ES. Análise do controle de temperatura dos alimentos servidos em Unidade de Alimentação e Nutrição universitária na cidade de Picos-PI, Brasil. Res, Soc Develop. 2019;8(2). doi: http://dx.doi.org/10.33448/rsd-v8i2.563.

32. Wietholter MJ, Fassina P. Temperaturas de armazenamento e distribuição dos alimentos. Rev Seg Alimentar Nutr. 2017;24(1):17-25.

33. Correa VG, et al. Monitoramento do binômio tempo e temperatura nos processos de produção de alimentos em um restaurante universitário. Braz J Food Res. 2017;8(2):46-56.

34. Giustina CLD, Venzke JG. Avaliação da temperatura dos alimentos distribuídos para os pacientes de um hospital escola do município de Porto Alegre-RS. Repositório Digital Lume UFRGS. 2019. 